Pacific Journal of Mathematics

ESSENTIAL TORI IN 4-MANIFOLD BOUNDARIES 


\title{
ESSENTIAL TORI IN 4-MANIFOLD BOUNDARIES
}

\author{
MARTIN SCHARLEMANN
}

The central result is an analogue for four manifolds of the loop theorem, in which, with suitable restrictions on $\pi_{2}$, essential loops in the boundary are replaced by essential tori.

A map $f: T^{2} \rightarrow X$ of a torus into a space is called essential if the induced map $f_{\#}: \pi_{1}\left(T^{2}\right) \rightarrow \pi_{1}(X)$ is injective [Jo]. A map which is not essential is called inessential. If $X$ is a 3-manifold and $f$ is an essential imbedding then $f\left(T^{2}\right)$ is incompressible in $X$. The question which we examine here is the following: Suppose $M$ is a 4-manifold and there is an essential map $f: T^{2} \rightarrow \partial M$ which is inessential in $M$. Is there an incompressible torus in $\partial M$ which is inessential in $M$ ?

The question may be viewed as a 4-dimensional analogue of the loop theorem of Papakyriakopoulos [Pa], which says that if there is an essential map of a circle to the boundary of a 3-manifold which is inessential in the 3-manifold, then an imbedding with this property exists. There are, however, two points to keep in mind about this comparison. In the classical case, the usefulness of the theorem is greatly enhanced by combining it with Dehn's lemma [St], for which no good 4-dimensional analogue has yet been found. Secondly, a map $f: S^{1} \rightarrow X$ is called essential if $f_{\#}: \pi_{1}\left(S^{1}\right) \rightarrow \pi_{1}(X)$ is merely non-trivial, not necessarily injective. Thus the meaning of the word "essential" has come to have slightly different meanings, depending on whether the domain of the map is a circle or a torus. However, in the former case, if the range $X$ is a surface or an orientable, sufficiently large 3-manifold the notions coincide, for $\pi_{1}(X)$ has no torsion.

Before attempting to answer the question, consider two classes of counterexamples. First, let $T$ be any orientable surface with $\chi(T)<0$, and $f: S^{1} \rightarrow T$ be a map not homotopic to a multiple of any imbedded loop. Then $\bar{f}=f \times \mathrm{id}_{S^{1}}: S^{1} \times S^{1} \rightarrow T \times S^{1} \times 0 \subset T \times S^{1} \times I$ is an essential torus in $T \times S^{1} \times I$. Let $Q$ be the 4-manifold obtained by adding a 2-handle to $T \times S^{1} \times I$ on an imbedded loop in $T \times S^{1} \times 1$ homotopic to $f\left(S^{1}\right) \times$ (point). Then $\bar{f}$ is essential in $T \times S^{1} \times 0$, inessential in $Q$, yet any incompressible torus in $T \times S^{1} \times 0$ remains essential in $Q$. Notice that the addition of the 2-handle generates a non-trivial element of $\pi_{2}$, just as adding a 2-disk across the meridian of a torus does.

A more subtle counterexample is the following: Let $T$ be a closed orientable surface with $\chi(T) \leq 0$ and $\varphi: T \rightarrow T$ be a periodic automorphism of period $p$ which is free except at a finite collection of points 
$x_{1}, \ldots, x_{n}$. The mapping torus $T_{\varphi}=T \times I /(x, 0)=(\varphi(x), 1)$ is then a closed 3-manifold whose $p$-fold cover is $T \times S^{1}$. Examples of such $T_{\varphi}$ can be obtained, for example, by doing 0 -framed surgery on torus knots.

Using polar coordinates $(r, \theta)$ for the $D^{2}$ factor, define $\bar{\varphi}: T \times D^{2} \rightarrow$ $T \times D^{2}$ by $\bar{\varphi}(x, r, \theta)=(\varphi(x), r, \theta+2 \pi / p)$. Then $\bar{\varphi}$ generates an effective $Z_{p}$ action on $T \times D^{2}$. The only points on which $\bar{\varphi}$ is not free are points of the form $\left(x_{i}, 0\right)$ in $T \times D^{2}$. Remove an invariant open tubular neighborhood of $\left\{\left(x_{i}, 0\right)\right\}$ from $T \times D^{2}$, and let $M$ be the quotient manifold. Then $T_{\varphi} \subset \partial M$, and for any essential map $f: S^{1} \rightarrow T$, the composition of $f \times \mathrm{id}_{\partial D^{2}}: S^{1} \times \partial D^{2} \rightarrow T \times \partial D^{2}$ with the projection to $\partial M$ will be a map which is essential in $T_{\varphi} \subset \partial M$ but not in $M$. Yet not only may $T_{\varphi}$ fail to have an incompressible torus which is inessential in $M$, but may fail to have any incompressible torus. In particular, after 0 -framed surgery on a torus knot not of type $(2,3)$ the resulting 3-manifold has no incompressible tori. Note here that $\pi_{2}(M)=\pi_{2}\left(T \times D^{2}\right)=0$.

Faced with such discouraging evidence, it is perhaps surprising that these two situations exhaust the counterexamples, that is, by limiting the size of $\pi_{2}\left(M^{4}\right)$ and eliminating a small class of 3-manifolds from consideration, our original question can be answered affirmatively. Explicitly, we say that $\pi_{i}(M), i \geq 2$, is small, if there is a subgroup $G \subseteq \pi_{1}(M)$ such that $G$ is of infinite index in its normalizer in $\pi_{1}(M)$ and $\pi_{i}(M) / G$ is finitely generated. Here $G$ acts on $\pi_{i}(M)$ by change of basepoint. A 3-manifold is unexceptional if it is compact, orientable, irreducible, connected, sufficiently large and not a Seifert fibered space over $S^{2}$ with precisely 3 exceptional fibers. We then have:

THEOREM 0. Suppose $N$ is an unexceptional 3-manifold lying in the boundary of a 4-manifold $M$ with $\pi_{2}(M)$ small. Then if there is an essential map $f: T^{2} \rightarrow N$ which is inessential in $M$, then there is an incompressible torus in $N$ which is inessential in $M$.

1. The exceptional 3-manifolds. Here we show the relation between the counterexamples with boundary $T_{\varphi}$ discussed above, and the requirement in Theorem 0 that $N$ not be a Seifert fibered space with precisely 3 exceptional fibers.

Suppose $N$ is an orientable, sufficiently large, compact 3-manifold which is a Seifert fibered space with projection $p: N \rightarrow S$.

LEMMA 1.1. If there is no incompressible torus in $N$ containing a regular fiber then $S$ is a sphere and $N$ has precisely 3 exceptional fibers.

Proof. If $S$ is nonorientable, $N$ contains an incompressible 1-sided Klein bottle whose regular neighborhood has boundary the required torus. If $S$ is orientable the center of $\pi_{1}(N)$ contains a free cyclic summand 
generated by a regular fiber [JS]. Suppose there is an imbedded loop $f$ : $S^{1} \hookrightarrow S$ inducing an injection of fundamental groups. Homotope $f$ so it intersects no exceptional points (i.e., images of exceptional fibers) in $S$. Then $p^{-1}\left(f\left(S^{1}\right)\right)$ is a torus $T$ in $N$, and the following diagram commutes

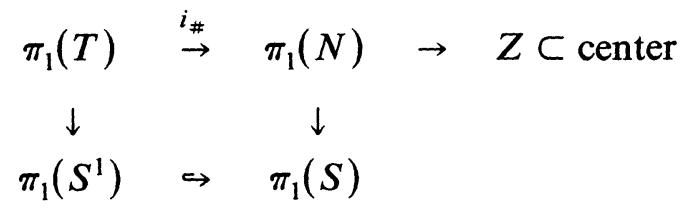

Thus $i_{\#}$ is injective and $T$ is incompressible.

Hence we may assume $S=R P^{2}$ or $S=S^{2}$. An easy calculation shows that an orientable Seifert bundle over $D^{2}$ with more than one exceptional orbit has incompressible boundary. Suppose $S=R P^{2}$, and remove the one-sided Klein bottle in $N$ covering a one-sided loop in $R P^{2}$. The fundamental group of the resulting Seifert bundle $N^{\prime}$ over $D^{2}$ injects into that of $N$, since the covering projection from the torus to the Klein bottle is injective. Hence, if $N$ has more than one exceptional orbit, $N^{\prime}$, hence $N$, contains the required torus. If there is at most one exceptional orbit $M$ is a prism manifold [Or], with universal covering space $S^{3}$, and thus not sufficiently large.

If $S=S^{2}$ and $M$ has four or more exceptional orbits, then it can be divided into two pieces, each a Seifert fibered space over a disk with two or more exceptional orbits. Their common boundary is the required torus. If there are two or fewer exceptional orbits, $M$ is a Lens space [Or] hence not sufficiently large. This proves 1.1 .

LEMMA 1.2. Let $p: N \rightarrow S^{2}$ be an orientable, sufficiently large Seifert fibered space with 3 exceptional orbits. Then there is a surface $T, a$ periodic automorphism $\varphi: T \rightarrow T$ such that $N=T_{\varphi}=T \times I /(x, 0)=(\varphi(x), 1)$.

Proof. Let $T$ be an incompressible surface in $N$. $T$ fails to separate $N$, for, if it did, $N$ could be written as the union along the boundary of two Seifert fibered spaces over disks with incompressible common boundary $T$ [He, Theorem 12.8]. But each piece would then have at least two exceptional orbits, a contradiction.

Since $T$ fails to separate $N, H_{1}(N)$ is infinite and $N=T_{\varphi}[\mathbf{H e}$, Theorem 12.6], for $\varphi: T \rightarrow T$ homotopic, hence isotopic [Ni], to a periodic automorphism. That the fibers of $T_{\varphi}$ correspond to those of $N$ follows from the uniqueness of Seifert fibrations for these manifolds [Or].

REMARK. In the first section we showed that if $N=T_{\varphi}$, for $\varphi: T \rightarrow T$ a periodic automorphism, then there is a 4-manifold $M$ with $N \subset \partial M$ such that $\operatorname{ker}\left(\pi_{1}(N) \rightarrow \pi_{1}(M)\right)$ is generated by the circle factor in the cover 
$T \times S^{1}$ of $T_{\varphi}=N$. Now we have seen that the exceptional 3-manifolds of 1.1 can be written as $T_{\varphi}$, but the circle factor in $T \times S^{1}$ is contained in no incompressible torus. This explains why we must exclude such $N$ from consideration.

2. Manifolds with small $\pi_{i}$. In this section we prove the one property of manifolds with small $\pi_{i}$ which is needed. Suppose $M$ is a manifold, with universal cover $\tilde{M}$, and suppose, for some $i \geq 2$, that $\pi_{l}(M)$ is small. Let $X \subset \tilde{M}$ be a compact set which does not separate $\tilde{M}$.

Proposition 2.1. Any map $f: S^{i} \rightarrow \tilde{M}$ is homotopic to a map into $\tilde{M}-X$.

Proof. By hypothesis there is a subgroup $G \subset \pi_{1}(M)$ of infinite index in its normalizer such that $\pi_{i}(M) / G$ is finitely generated. Let $M_{G}$ denote the cover of $M$ corresponding to $G$, and $p_{G}: \tilde{M} \rightarrow M_{g}$ the projection. There are a finite collection of maps $\left\{\beta_{j}: S^{i} \rightarrow M_{G}\right\}, 1 \leq j \leq n$, generating $\pi_{i}\left(M_{G}\right)$ as a $G$-module.

Since $X$ is compact, there are only a finite number of covering translations $T: M_{G} \rightarrow M_{G}$ for which, for some $j, T\left(X_{G}\right) \cap \beta_{j}\left(S^{i}\right) \neq \varnothing$. Then, since $G$ is of infinite index in its normalizer, there is a $T: M_{G} \rightarrow M_{G}$ such that for all $1 \leq j \leq n, T p_{G}(X) \cap \beta_{j}\left(S^{i}\right)=\varnothing$.

Now $T p_{G} f: S^{i} \rightarrow M_{G}$ is homotopic to a map into a set consisting of $\cup_{j=1}^{n} \beta_{j}\left(S^{i}\right)$ and (by compactness of $\left.S^{i}\right)$ a finite collection of arcs running from a base point of $M_{G}$ to the $\beta_{j}\left(S^{i}\right)$. In fact, there is a simply connected space $Y$ consisting of a finite collection of $i$-spheres together with arcs joining basepoints to a common point, such that $T p_{G} f$ is homotopic to a map which factors through $Y$ and the images of the $i$-spheres of $Y$ in $M_{G}$ are disjoint from $T p_{G}(X)$. Applying $T^{-1}$ and lifting, the map $f: S^{1} \rightarrow \bar{M}$ is homotopic to a map factoring through a map $f^{\prime}: Y \rightarrow \tilde{M}$ which maps the $i$-spheres of $Y$ into $\tilde{M}-X$. Since $\tilde{M}-X$ is connected and $\tilde{M}$ is 1 -connected, $f^{\prime}$ is homotopic to a map into $\tilde{M}-X$. Then so is $f$.

3. A special case. In this section we consider a special case of Theorem 0 in which the relation between the problem at hand and the loop theorem is crystallized. Let $N$ be the product of an orientable surface $S$ and the circle $S^{1}$, and assume that an essential torus, inessential in $M$, is given by the product of an essential map $\alpha: S^{1} \rightarrow S$ and the identity on the $S^{1}$ factor. We further assume that the kernel of $\left(\alpha \times \mathrm{id}_{S^{1}}\right)_{\#}$ : $\pi_{1}\left(S^{1} \times S^{1}\right) \rightarrow \pi_{1}(M)$ is generated by $\alpha: S^{1} \rightarrow S \times q, q$ in $S^{1}$.

Notice that if $\chi(S) \geq 0$ there is an incompressible torus in $N=S \times S^{1}$ which is inessential in $M$. Indeed, since $S$ is the torus or annulus, $\alpha$ itself is homotopic to a multiple of an imbedded circle. 
Suppose now $\chi(S)<0$ and $L$ is any non-empty normal subgroup of $\pi_{1}(S \times q)$ not containing $\alpha$. Then we have

Proposition 3.1. Under the above assumptions, there is an imbedding $\bar{\alpha}: S^{1} \rightarrow S \times q$ such that, for some $i>0, \bar{\alpha}^{i}$ is null-homotopic in $M$, but does not represent an element of $L$.

The proof will use two lemmas on surfaces appearing, for example, in [Th]. Suppose $S$ is a geodesically complete compact surface of constant negative curvature.

LeMma 3.2. Any map $\alpha: S^{1} \rightarrow S$ is homotopic to a geodesic map.

Proof. Let $\tilde{S}$ denote the universal cover of $S$, imbedded in the hyperbolic plane regarded as the interior of $D^{2}$. Corresponding to $\alpha$ is a covering translation $T: \tilde{S} \rightarrow \tilde{S}$. Let $\tilde{\alpha}$ be defined so that

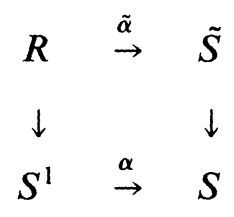

commutes. Then $T$ maps $\tilde{\alpha}(R)$ to itself. Define $\alpha_{-}$and $\alpha_{+}$in $\partial D^{2}$ by $\alpha_{ \pm}=\lim _{t \rightarrow \pm \infty} \tilde{\alpha}(t)$. Let $\tilde{\alpha}_{0}: R \rightarrow \tilde{S}$ be the unique geodesic connecting $\alpha_{-}$ and $\alpha_{+}$. Clearly, $T\left(\alpha_{-}\right)=\alpha_{-}, T\left(\alpha_{+}\right)=\alpha_{+}$, so $T \tilde{\alpha}_{0}(R)=\tilde{\alpha}_{0}(R)$. The quotient of $\tilde{\alpha}_{0}(R)$ by the action of $T$ is then the required map $\alpha_{0}: S^{1} \rightarrow \tilde{S}$.

LEMMA 3.3. For any $l \in R$, there are only a finite number of geodesic maps $S^{1} \rightarrow S$ of length less than $l$.

Proof. By compactness, it suffices to show there are only finitely many which pass through a given coordinate chart $U \subset S$. In $\tilde{S}$, the universal cover of $S$ in the hyperbolic plane, there are only a finite number of lifts of $U$ within a distance $l$ of a given lift $\tilde{U}_{0}$ of $U$. Hence it suffices to show that there are only a finite number of geodesics in $\tilde{S}$ which intersect two given lifts $\tilde{U}_{0}, \tilde{U}_{1}$ and are invariant under the covering translation $T$ : $\tilde{S} \rightarrow \tilde{S}$ carrying $\tilde{U}_{0}$ to $\tilde{U}_{1}$. In fact there is exactly one such geodesic. For suppose $x_{0}, y_{0} \in \tilde{U}_{0}$ and there are distinct geodesics in $\tilde{S}$ running from $x_{0}$ to $T\left(x_{0}\right), y_{0}$ to $T\left(y_{0}\right)$. Connect $x_{0}$ to $y_{0}$ by another geodesic in $\tilde{U}_{0}$, and $T\left(x_{0}\right)$ to $T\left(y_{0}\right)$ by its translate under $T$. This produces a quadrilateral in $\tilde{S}$ with geodesic sides and interior angles summing to $2 \pi$, contradicting the Gauss-Bonnet theorem. This proves the lemma. 
To prove 3.1, choose a geodesically complete metric on $S$ of constant negative curvature. Let $\delta$ denote the collection of all geodesic maps $S^{1} \rightarrow S \times q$ which are null-homotopic in $M$, do not represent an element of $L \subset \pi_{1}(S)$ and are of minimal length $l$. Lemmas 3.2, 3.3 and the hypothesis of 3.1 assure that $\mathcal{S}$ is non-empty. Lemma 3.3 assures that $\mathcal{S}$ is finite. We intend to show that, under the hypotheses of $3.1, \mathcal{S}$ consists solely of multiples of imbedded loops. This is equivalent to showing that any element of $\mathcal{S}$ contains no transverse self-intersections.

Let $p: \tilde{M} \rightarrow M$ denote the universal cover of $\tilde{M}, \hat{N}$ denote $p^{-1}(N)$ and $\hat{S}$ denote $p^{-1}(S)$ respectively. Note, that by hypothesis $\hat{S} \neq \hat{S}$, for any element of $\mathcal{S}$ lifts to an essential loop in $\hat{S}$. Let $\alpha: S^{1} \rightarrow S \times q$ be in $\mathcal{S}$, and $\hat{\alpha}$ a lift of $\alpha$ to $\hat{S}$.

Notation. For $f: S^{1} \rightarrow M, \theta_{0} \neq \theta_{1}$ in $S^{1}$, let $f\left[\theta_{0}, \theta_{1}\right]$ denote the image of the segment in $S^{1}$ running counterclockwise from $\theta_{0}$ to $\theta_{1}$ and $f^{-1}\left[\theta_{0}, \theta_{1}\right]$ denote the image of the segment from $\theta_{0}$ to $\theta_{1}$ running counterclockwise. Products of segments are composed from left to right, i.e., $f\left[\theta_{0}, \theta_{1}\right] \cdot f\left[\theta_{1}, \theta_{2}\right]=f\left[\theta_{0}, \theta_{2}\right]$.

\section{LEMMA 3.4. $\hat{\alpha}$ is an imbedding.}

Proof. If not, there are $\theta_{0} \neq \theta_{1}$ in $S^{1}$ such that $\hat{\alpha}\left(\theta_{0}\right)=\hat{\alpha}\left(\theta_{1}\right)$. Then $\hat{\alpha} \mid\left[\theta_{0}, \theta_{1}\right]$ and $\hat{\alpha} \mid\left[\theta_{1}, \theta_{0}\right]$ factor through maps $\alpha_{1}: S^{1} \rightarrow \tilde{M}, \alpha_{2}: S^{1} \rightarrow \tilde{M}$ respectively such that $\alpha_{1} \cdot \alpha_{2}=\hat{\alpha}$. Since $\pi_{1}(\tilde{M})=0, p\left(\alpha_{1}\right)$ and $p\left(\alpha_{2}\right)$ are null-homotopic in $M$ and are clearly both of length less than $l$, the length of $\alpha$. Furthermore, since $\alpha$ is not in $L$, some $p_{i}(\alpha)$ is not in $L$. By 3.2, there is a geodesic homotopic to (and of shorter length than) $p_{t}(\alpha)$. This contradicts the definition of $\mathfrak{S}$.

LEMma 3.5. If $\hat{\alpha}_{0}, \hat{\alpha}_{1}: S^{1} \rightarrow M$ are lifts of $\alpha$ such that $\hat{\alpha}_{0}\left(S^{1}\right) \neq \hat{\alpha}_{1}\left(S^{1}\right)$ then the $\hat{\alpha}_{t}$ intersect transversally in $\hat{S}$ with algebraic intersection zero.

Proof. If $\hat{\alpha}_{0}\left(S^{1}\right)$ and $\hat{\alpha}_{1}\left(S^{1}\right)$ intersect non transversally then, since they are geodesics, $\hat{\alpha}_{0}\left(S^{1}\right)=\hat{\alpha}_{1}\left(S^{1}\right)$. This verifies the first claim.

By hypothesis, $\alpha \times \mathrm{id}_{S^{1}}: S^{1} \times S^{1} \rightarrow N$ lifts to a map $\beta: S^{1} \times R \rightarrow \hat{N}$ which restricts to $\hat{\alpha}_{0}$ on $S^{1} \times\{0\}$. Since $\hat{\alpha}_{0}$ is null-homotopic in $\tilde{M}$, it extends to a map $\bar{\alpha}_{0}: D^{2} \rightarrow \tilde{M}$. A translate of $\bar{\alpha}_{0}$ provides a map $\bar{\alpha}_{1}$ : $D^{2} \rightarrow \tilde{M}$ extending $\hat{\alpha}_{1}$.

Let $Q$ denote the complement in $D^{2} \times R$ of small open balls about $\left\{(0,(2 i+1) / 2) \mid 0 \in D^{2}, i \in Z\right\}$. Then $\partial Q=S^{1} \times R$ together with a countable collection of 2 -spheres. Translates of $\hat{\alpha}_{0}$ also produce a proper map $\bar{\beta}: Q \rightarrow \tilde{M}$ extending $\beta$. By 2.1 , we may homotope the 2 -sphere components of $\partial Q$ so that $\bar{\beta}$ maps them to $\tilde{M}-\bar{\alpha}_{1}\left(D^{2}\right)$. Put $\bar{\beta}$ and $\bar{\alpha}_{1}$ in 
general position by a small homotopy. Then $\bar{\beta}(Q) \cap \bar{\alpha}_{1}\left(D^{2}\right)$ provides a null homology of $\hat{\alpha}_{0}\left(S^{1}\right) \cap \hat{\alpha}_{1}\left(S^{1}\right)$, proving 3.5.

Suppose now that $\alpha$ has transverse self-intersections. According to 3.4 and 3.5 there are, in $\hat{S} \subset \partial M$, two distinct lifts $\hat{\alpha}_{0}: S^{1} \rightarrow \hat{S}$ and $\hat{\alpha}_{1}: S^{1} \rightarrow \hat{S}$ which intersect transversally at least once but algebraically intersect trivially. Thus there are at least two pairs of points $\theta_{0} \neq \theta_{1}, \varphi_{0} \neq \varphi_{1}$ in $S^{1}$ such that $\hat{\alpha}_{0}\left(\theta_{0}\right)=\hat{\alpha}_{1}\left(\theta_{1}\right), \hat{\alpha}_{0}\left(\varphi_{0}\right)=\hat{\alpha}_{1}\left(\varphi_{1}\right)$.

Let $l_{1}, l_{2}, l_{3}, l_{4}$ denote the length of the arcs $\alpha\left[\theta_{0}, \varphi_{0}\right], \alpha\left[\varphi_{0}, \theta_{0}\right]$, $\alpha\left[\theta_{1}, \varphi_{1}\right], \alpha\left[\varphi_{1}, \theta_{1}\right]$ respectively. Since $\alpha \in \mathcal{S}, l_{1}+l_{2}=l_{3}+l_{4}=l$. Then one of each pair $\left\{l_{1}+l_{3}, l_{2}+l_{4}\right\}\left\{l_{1}+l_{4}, l_{2}+l_{3}\right\}$ is no greater than $l$. With no loss of generality, assume $l_{1}+l_{3} \leq l$.

Let

$$
\begin{aligned}
\gamma_{0} & =\hat{\alpha}_{1}\left[\theta_{1}, \varphi_{1}\right] \hat{\alpha}_{0}\left[\varphi_{0}, \theta_{0}\right], \\
\gamma_{1} & =\hat{\alpha}_{1}^{-1}\left[\theta_{1}, \varphi_{1}\right] \hat{\alpha}_{0}^{-1}\left[\varphi_{0}, \theta_{0}\right], \\
\gamma_{2} & =\hat{\alpha}_{0}\left[\theta_{0}, \varphi_{0}\right] \hat{\alpha}_{1}^{-1}\left[\varphi_{1}, \theta_{1}\right] .
\end{aligned}
$$

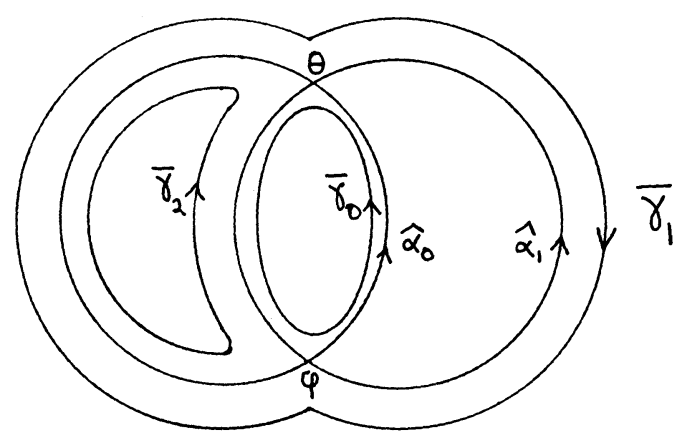

Let $\bar{\gamma}_{i}$ denote a geodesic homotopic to $\gamma_{i}, 0 \leq i \leq 2$. Since $\hat{\alpha}_{0}$ and $\hat{\alpha}_{1}$ intersect transversally the length of each $\bar{\gamma}_{i}$ is less than that of $\gamma_{i}$. Furthermore, since the length of $\gamma_{2}$ is $l_{1}+l_{3} \leq l$ and the lengths of $\gamma_{0}$ and $\gamma_{1}$ are $l_{2}+l_{3}$ and $l_{1}+l_{4}$ respectively, we know the lengths of $\bar{\gamma}_{2}$ and one of $\bar{\gamma}_{i}, i=0,1$, are less than $l$. By definition of $l, \bar{\gamma}_{2}$ and $\bar{\gamma}_{i}$ project to elements of $L$. But $\bar{\gamma}_{2} \cdot \bar{\gamma}_{0}$ and $\bar{\gamma}_{1} \cdot \bar{\gamma}_{2}$ are homotopic to $\hat{\alpha}_{0}$ and $\hat{\alpha}_{1}$ respectively, which do not project to elements of $L$. The contradiction completes the proof.

4. The general case. Our strategy here is to reduce the proof of Theorem 0 to the special case of $\S 3$. The torus theorem of Jaco-Shalen, Johannson, and Waldhausen [JS], [Jo], [Wa] states that any essential map $f: T^{2} \rightarrow N$, for $N$ connected, orientable, irreducible and sufficiently large, 
is homotopic to a map into a Seifert fibered space, with incompressible boundary components, contained in $N$. We may, therefore, assume that $N$ is a Seifert fibered space.

Let $F$ be the fiber of $N, q: N \rightarrow N / F=S$ be the projection, and $\beta$ in $\pi_{1}(N)$ be the class represented by $F$. By hypothesis, $N$ is not a Seifert fibered space over $S^{2}$ with 3 exceptional fibers, so, by 1.1, there is an incompressible torus in $N$ containing a fiber $F$. Then if, for some $i \neq 0, \beta^{i}$ is trivial in $M$, we are finished. We henceforth assume, therefore:

$\beta$ generates a free cyclic subgroup of $\pi_{1}(M)$.

Although $N$ is assumed orientable, $S$ may not be, for a one-sided circle $C$ in $S$ might be covered by a one-sided Klein bottle $K$ in $N$. But suppose $f: T^{2} \rightarrow N$ is essential in $N$, and $\alpha: S^{1} \rightarrow N$ represents a non-trivial element of

$$
\operatorname{ker}\left(f_{\#}\left(\pi_{1}\left(T^{2}\right)\right) \rightarrow \pi_{1}(M)\right) .
$$

Clearly, the centralizer of $\alpha$ in $\pi_{1}(N)$ contains $f_{\#}\left(\pi_{1}\left(T^{2}\right)\right)$ and so is not cyclic. By $[\mathbf{J S}], q(\alpha)$ is an orientation preserving loop in $S$, and, therefore, may be homotoped off $C$. It follows that $\alpha$ may be homotoped off $K$. It suffices to assume, therefore, that $S$ is orientable. Now homotope $\alpha$ so that $q(\alpha)$ is in general position and disjoint from the exceptional points of $S$ (i.e., the images of the exceptional fibers in $N$ ). Remove from $S$ small open disks around each exceptional point and also around two ordinary points $p_{1}, p_{2}$, chosen so that some are between $p_{1}$ and $p_{2}$ intersects $q(\alpha)$ transversally exactly $N_{0}=q^{-1}\left(S_{0}\right)$.

The removal of all these orbits reduces $N_{0}$ to a trivial bundle over $S_{0}$. Moreover, our choice of $p_{1}$ and $p_{2}$ ensures that there is a bundle trivialization $h: N_{0} \rightarrow S_{0} \times S^{1}$ such that $h(\alpha)$ is homotopic to $q(\alpha) \times p$, for some point $p$ in $S^{1}$.

Let $L \subset \pi_{1}\left(S_{0} \times p\right)$ denote the normal subgroup generated by loops around $p_{1}$ and $p_{2}$ in $S_{0} \times p$. Since each such loop is homotopic in $N$ to some power of $\beta$, and, by assumption $(*), \alpha$ is not homotopic to any power of $\beta$, it follows that $q(\alpha) \times p$ is not in $L$. By 3.1 there exist $\bar{\alpha} \in \pi_{1}\left(S_{0}\right)$ and $i>0$ such that $\bar{\alpha}$ is representable by an imbedding $\bar{\alpha}$ : $S^{1} \rightarrow S_{0}, h_{\#}^{-1}\left(\bar{\alpha}^{i}\right)$ is trivial in $\pi_{1}(M)$ and $\bar{\alpha}^{i}$ is disjoint from $L$. The proof of Theorem 0 is completed by verifying the following

Claim. $h^{-1}\left(\bar{\alpha} \times \operatorname{id}_{S^{1}}\right): S^{1} \times S^{1} \rightarrow N_{0} \subset N$ is incompressible in $N$.

Proof of claim. As in the proof of 1.1, if $q h^{-1} \bar{\alpha}$ is essential in $S$, then the torus is essential, hence incompressible, in $N$.

If $q h^{-1} \bar{\alpha}$ is inessential in $S$ it bounds a disk containing points which were deleted in constructing $S_{0}$. Such a disk contains at least one image of 
an exceptional fiber, for otherwise $\bar{\alpha}$ would be in $L$. If each such disk contains the image of more than one exceptional fiber then, again as in 1.1 , the torus is incompressible.

So suppose $q h^{-1} \bar{\alpha}$ contains the image $p_{0}$ of exactly one exceptional fiber. Let $\gamma$ denote a loop around $p_{0}$ in a cross-section of $N$ near $q^{-1}\left(p_{0}\right)$ given by the standard description of $N$ as a Seifert fibered space (see e.g., [Or]). Then $h^{-1}(\bar{\alpha})=\gamma \beta^{r}$ for some $r$, determined by the trivialization $h$ chosen above. Let $(s, t)$ be the Seifert invariants of the fiber over $p_{0}$, $0<t<s$. Then $\gamma^{s} \beta^{t}=1$ in $\pi_{1}(N)$ so $h^{-1}\left(\bar{\alpha}^{s i}\right)=\gamma^{s i} \beta^{r s i}=\beta^{(r s-t) i}$ is trivial in $\pi_{1}(M)$, contradicting $(*)$ and thus proving the theorem.

\section{REFERENCES}

[He] J. Hempel, 3-Manifolds, Ann. Math. Study no. 86, Princeton Univ. Press, 1976.

[JS] W. Jaco and P. Shalen, Seifert fibered spaces in 3-manifolds, Rice University, 1977.

[Jo] K. Johannson, Équivalences d'homotopie des variétes de dimension 3, C. R. Acad. Sci. Paris, 66 (1975), 1009-1010.

[Ni] J. Nielsen, Abbildungsklassen endlicher Ordnung, Acta Math., 75 (1942), 23-115.

[Or] P. Orlik, Seifert manifolds, Lecture notes in mathematics no. 291, Springer, 1972.

[Pa] C. D. Papakyriakopoulos, On solid tori, Proc. London Math. Soc., 7 (1957), 281-299.

[St] J. R. Stallings, On the loop theorem, Ann. Math., 72 (1960), 12-19.

[Th] W. Thurston, Geometric topology seminar notes, Princeton Univ. 1975.

[Wa] F. Waldhausen, On the determination of some bounded 3-manifolds by their fundamental groups alone, Proceedings of the International Symposium on Topology and its applications, Herceg-Novi, Yugoslavia, 1968: Beograd (1969), 331-332.

Received March 28, 1979. Supported in part by an NSF grant.

UNIVERSITY OF CALIFORNIA

SANTA Barbara, CA 93106 



\title{
PACIFIC JOURNAL OF MATHEMATICS EDITORS
}

\author{
Donald BabBitT (Managing Editor) \\ University of California \\ Los Angeles, CA 90024 \\ Hugo Rossi \\ University of Utah \\ Salt Lake City, UT 84112 \\ C. C. Moore and Arthur Ogus \\ University of California \\ Berkeley, CA 94720
}

J. DugundiI

Department of Mathematics

University of Southern California

Los Angeles, CA 90089-1113

R. FINN and H. SAMELSON

Stanford University

Stanford, CA 94305

\section{ASSOCIATE EDITORS}
R. ARENS
E. F. BECKENBACH
B. H. NeUMANN
F. WOLF
K. YosHIDA

(1906-1982)

\section{SUPPORTING INSTITUTIONS}

UNIVERSITY OF ARIZONA

UNIVERSITY OF BRITISH COLUMBIA

CALIFORNIA INSTITUTE OF TECHNOLOGY

UNIVERSITY OF CALIFORNIA

MONTANA STATE UNIVERSITY

UNIVERSITY OF NEVADA, RENO

NEW MEXICO STATE UNIVERSITY

OREGON STATE UNIVERSITY

\author{
UNIVERSITY OF OREGON \\ UNIVERSITY OF SOUTHERN CALIFORNIA \\ STANFORD UNIVERSITY \\ UNIVERSITY OF HAWAII \\ UNIVERSITY OF TOKYO \\ UNIVERSITY OF UTAH \\ WASHINGTON STATE UNIVERSITY \\ UNIVERSITY OF WASHINGTON
}

The Supporting Institutions listed above contribute to the cost of publication of this Journal, but they are not owners or publishers and have no responsibility for its content or policies.

Mathematical papers intended for publication in the Pacific Journal of Mathematics should be in typed form or offset-reproduced (not dittoed), double spaced with large margins. Please do not use built up fractions in the text of the manuscript. However, you may use them in the displayed equations. Underline Greek letters in red, German in green, and script in blue. The first paragraph must be capable of being used separately as a synopsis of the entire paper. In particular it should contain no bibliographic references. Please propose a heading for the odd numbered pages of less than 35 characters. Manuscripts, in triplicate, may be sent to any one of the editors. Please classify according to the scheme of Math. Reviews, Index to Vol. 39. Supply name and address of author to whom proofs should be sent. All other communications should be addressed to the managing editor, or Elaine Barth, University of California, Los Angeles, California 90024.

There are page-charges associated with articles appearing in the Pacific Journal of Mathematics. These charges are expected to be paid by the author's University, Government Agency or Company. If the author or authors do not have access to such Institutional support these charges are waived. Single authors will receive 50 free reprints; joint authors will receive a total of 100 free reprints. Additional copies may be obtained at cost in multiples of 50 .

The Pacific Journal of Mathematıcs is issued monthly as of January 1966. Regular subscription rate: $\$ 132.00$ a year (6 Vol., 12 issues). Special rate: $\$ 66.00$ a year to individual members of supporting institutions.

Subscriptions, orders for numbers issued in the last three calendar years, and changes of address should be sent to Pacific Journal of Mathematics, P.O. Box 969, Carmel Valley, CA 93924, U.S.A. Old back numbers obtainable from Kraus Periodicals Co., Route 100, Millwood, NY 10546.

The Pacific Journal of Mathematics ISSN 0030-8730 is published monthly by the Pacific Journal of Mathematics at P.O. Box 969, Carmel Valley, CA 93924. Application to mail at Second-class postage rates is pend ing at Carmel Valley, California, and additional mailing offices. Postmaster: Send address changes to Pacific Journal of Mathematics, P. O. Box 969, Carmel Valley, CA 93924.

PUBLISHED BY PACIFIC JOURNAL OF MATHEMATICS, A NON-PROFIT CORPORATION

Copyright $(1) 1983$ by Pacific Journal of Mathematics 


\section{Pacific Journal of Mathematics}

\section{Vol. 105, No. $2 \quad$ October, 1983}

Spiros Argyros, On compact spaces without strictly positive measure . . . . . 257

Steven Robert Bell, Regularity of the Bergman projection in certain nonpseudoconvex domains ............................273

Carlos R. Borges and Gary Fred Gruenhage, Sup-characterization of

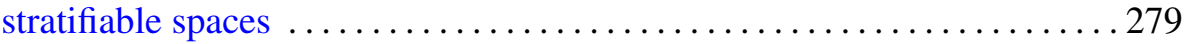

Giuseppe Ceresa and Alberto Collino, Some remarks on algebraic

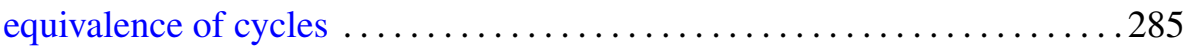

Charles Kam-Tai Chui and Maurice Hasson, Degree of uniform approximation on disjoint intervals ....................... 291

Gary Gundersen, Meromorphic functions that share two finite values with

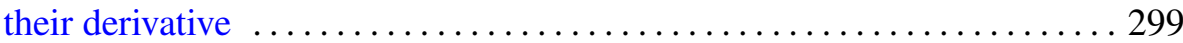

Richard I. Hartley, Lifting group homomorphisms ............... 311

Gerald William Johnson and David Lee Skoug, Notes on the Feynman integral. III. The Schroedinger equation ..................... 321

John Cronan Kieffer, Some topologies on the set of discrete stationary

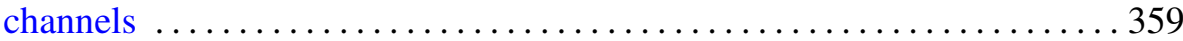

Harald Luschgy and Wolfgang Thomsen, Extreme points in the Hahn-Banach-Kantorovič setting . ............................... 387

Zbigniew Piotrowski, A. Rosłanowski and Brian M. Scott, The pinched-cube topology ................................ 399

Elias Saab and Paulette Saab, A dual geometric characterization of Banach

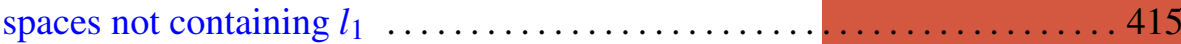

Walter Schachermayer, Norm attaining operators on some classical Banach

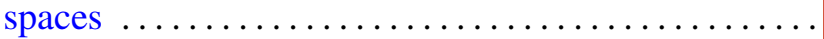

Martin Scharlemann, Essential tori in 4-manifold boundaries

Jacques C. H. Simon, Nonlinear representations of Poincaré group and global solutions of relativistic wave equations ..........

Adrian R. Wadsworth, $p$-Henselian field: $K$-theory, Galois cohomology, and graded Witt rings $\ldots \ldots \ldots \ldots \ldots \ldots \ldots \ldots \ldots$ 